\title{
Boxicity of Circular Arc Graphs
}

\author{
Diptendu Bhowmick ${ }^{*}$ L. Sunil Chandran ${ }^{\dagger}$
}

\begin{abstract}
A $k$-dimensional box is the cartesian product $R_{1} \times R_{2} \times \cdots \times R_{k}$ where each $R_{i}$ is a closed interval on the real line. The boxicity of a graph $G$, denoted as box $(G)$, is the minimum integer $k$ such that $G$ can be represented as the intersection graph of a collection of $k$-dimensional boxes: that is two vertices are adjacent if and only if their corresponding boxes intersect. A circular arc graph is a graph that can be represented as the intersection graph of arcs on a circle.

Let $G$ be a circular arc graph with maximum degree $\Delta$. We show that if $\Delta<$ $\left\lfloor\frac{n(\alpha-1)}{2 \alpha}\right\rfloor, \alpha \in \mathbb{N}, \alpha \geq 2$ then $\operatorname{box}(G) \leq \alpha$. We also demonstrate a graph with boxicity $>\alpha$ but with $\Delta=n \frac{(\alpha-1)}{2 \alpha}+\frac{n}{2 \alpha(\alpha+1)}+(\alpha+2)$. So the result cannot be improved substantially when $\alpha$ is large.

Let $r_{\text {inf }}$ be minimum number of arcs passing through any point on the circle with respect to some circular arc representation of $G$. We also show that for any circular arc graph $G, \operatorname{box}(G) \leq r_{i n f}+1$ and this bound is tight.

Given a family of $\operatorname{arcs} F$ on the circle, the circular cover number $L(F)$ is the cardinality of the smallest subset $F^{\prime}$ of $F$ such that the arcs in $F^{\prime}$ can cover the circle. Maximum circular cover number $L_{\max }(G)$ is defined as the maximum value of $L(F)$ obtained over all possible family of $\operatorname{arcs} F$ that can represent $G$. We will show that if $G$ is a circular arc graph with $L_{\max }(G)>4$ then $\operatorname{box}(G) \leq 3$.
\end{abstract}

Key words: Boxicity, circular arc graph.

\section{Introduction}

Let $\mathcal{F}$ be a family of non-empty sets. An undirected graph $G$ is the intersection graph of $\mathcal{F}$ if there exists a one-one correspondence between the vertices of $G$ and the sets in $\mathcal{F}$ such that two vertices in $G$ are adjacent if and only if the corresponding sets have non-empty intersection. If $\mathcal{F}$ is a family of intervals on the real line, then $G$ is called an interval graph.

A $k$-dimensional box or $k$-box is the cartesian product $R_{1} \times R_{2} \times \cdots \times R_{k}$, where each $R_{i}$ is a closed interval on the real line. The boxicity of a graph $G$ is defined to be the minimum integer $k$ such that $G$ is the intersection graph of a collection of $k$-boxes. Since 1-boxes are nothing but closed intervals on the real line, interval graphs are the graphs having boxicity 1 .

\footnotetext{
${ }^{*}$ Computer Science and Automation Department, Indian Institute of Science, Bangalore- 560012 Email: diptendubhowmick@gmail.com

${ }^{\dagger}$ Computer Science and Automation Department, Indian Institute of Science, Bangalore- 560012 Email: sunil@csa.iisc.ernet.in
} 
The concept of boxicity was introduced by F. S. Roberts [7] in 1969. Boxicity finds applications in fields such as ecology and operations research: It is used as a measure of the complexity of ecological [12] and social [10] networks and has applications in fleet maintenance [11]. Boxicity has been investigated for various classes of graphs [5] [13] 14] and has been related with other parameters such as treewidth [15] and vertex cover [16]. Computing the boxicity of a graph was shown to be NP-hard by Cozzens [9]. This was later strengthened by Yannakakis [6], and finally by Kratochvil [8] who showed that deciding whether boxicity of a graph is at most two itself is NP-complete. Recently Chandran et al [17] showed that for any graph $G$, box $(G) \leq \chi\left(G^{2}\right)$ where $G^{2}$ is the square of graph $G$ and $\chi$ is the chromatic number of a graph. From this they inferred that $\operatorname{box}(G) \leq 2 \Delta^{2}+2$, where $\Delta$ is the maximum degree of $G$. Very recently this result was improved by Esperet [18, who showed that box $(G) \leq \Delta^{2}+2$. In [19] Chandran et al have shown that $\operatorname{box}(G) \leq\lceil(\Delta+2) \log n\rceil$ where $\mathrm{n}$ is the number of vertices in $G$.

\section{Remark on previous approach to boxicity of circular arc graphs:}

In [15] it has been shown that for any graph $G$, box $(G) \leq \operatorname{treewidth}(G)+2$. If $G$ is circular arc graph then $\operatorname{treewidth}(G) \leq 2 \omega(G)-1$. It follows that box $(G) \leq 2 \omega(G)+1$. But the results shown in this paper are much stronger.

\section{$1.1 \quad$ Our results}

A graph $G$ is called a circular arc graph if it is the intersection graph of a collection of arcs on the circle that is, each vertex of this graph can be associated to an arc on a circle such that two vertices are adjacent if and only if the arcs corresponding to these vertices intersect. Circular arc graphs were first discussed as a natural generalization of interval graphs and they have since been studied extensively. See Golumbic [1 for a brief introduction and references on circular arc graphs. It is easy to see that a circular arc representation of an undirected graph $G$ which fails to cover some point $P$ on the circle will be topologically the same as an interval representation of $G$.

Since the definitions of interval graphs and circular arc graphs are quite similar, one may tend to conjecture that the boxicity of circular arc graphs may not be very high. But it comes as a surprise that the graph that achieves the highest boxicity belongs to the class of circular arc graphs! It was shown by Roberts [7] in his pioneering paper that the highest possible value of boxicity namely $\frac{n}{2}$ (Let $\mathrm{n}$ be an even number) was achieved by $\overline{\left(\frac{n}{2}\right) K_{2}}$, the complement of a perfect matching on $\mathrm{n}$ vertices. (Note that this graph is same as the complete $\frac{n}{2}$ partite graph on $\mathrm{n}$ vertices with each partition containing exactly two vertices). It turns out that $\overline{\left(\frac{n}{2}\right) K_{2}}$ is a circular arc graph. A circular arc representation of $\overline{\left(\frac{n}{2}\right) K_{2}}$ is given below. In this paper we will show that boxicity of a circular arc graph can be large only when its maximum degree is very high.

\section{A circular arc representation of $\overline{\left(\frac{n}{2}\right) K_{2}}$ :}

Let us take a circle. Place $\mathrm{n}$ points on the circle such that the distance between any adjacent pair of points is equal. Let these points be $p_{0}, p_{1}, \ldots, p_{n-1}$ in clockwise direction. Draw an arc from $p_{i}$ to $p_{\left(i+\frac{n}{2}-1\right) \bmod n}$ in clockwise direction for $0 \leq i<n$. It is easy to verify that the arc starting from $p_{i}$ is adjacent to all other arcs except the arc starting from $p_{\left(i+\frac{n}{2}\right) \bmod n}$. Thus this family of arcs gives a circular arc representation for $\overline{\left(\frac{n}{2}\right) K_{2}}$. 
Note that the vertices corresponding to the arcs starting from $p_{i}$ and $p_{\left(i+\frac{n}{2}\right)} \bmod n$ belong to the same partition in $\overline{\left(\frac{n}{2}\right) K_{2}}$ for $0 \leq i<\frac{n}{2}$, when we visualize $\overline{\left(\frac{n}{2}\right) K_{2}}$ as a complete $\frac{n}{2}$ partite graph.

Let $G$ be a circular arc graph on $\mathrm{n}$ vertices and with maximum degree $\Delta$. In this paper we will show the following results:

Result 1. If $\Delta<\left\lfloor n\left(\frac{\alpha-1}{2 \alpha}\right)\right\rfloor$ then $\operatorname{box}(G) \leq \alpha, \alpha \in \mathbb{N}, \alpha \geq 2$.

Remark: We are unable to show that the range of $\Delta$ as given by the above result, for which $\operatorname{box}(G) \leq \alpha$, is tight. But we can construct a circular arc graph with $\Delta=n \frac{(\alpha-1)}{2 \alpha}+\frac{n}{2 \alpha(\alpha+1)}+(\alpha+2)$ and box $(G)>\alpha$. Thus the result cannot be substantially improved when $\alpha$ is large.

Result 2. Given a family of $\operatorname{arcs} F$ on the circle, $r_{i n f}(F)$ is the minimum number of arcs passing through any point on the circle. Given a circular arc graph $G$, $r_{\text {inf }}(G)=\min r_{\text {inf }}(F)$ over all possible family of arcs $F$ that can represent $G$. We will show that $\operatorname{box}(G) \leq r_{\text {inf }}(G)+1$ and this bound is tight.

Result 3. Given a family of $\operatorname{arcs} F$ on the circle, the circular cover number $L(F)$ is the cardinality of the smallest subset $F^{\prime}$ of $F$ such that the arcs in $F^{\prime}$ can cover the circle. Maximum circular cover number $L_{\max }(G)$ is defined as the maximum value of $L(F)$ obtained over all possible family of $\operatorname{arcs} F$ that can represent $G$. We will show that if $G$ is a circular arc graph with $L_{\max }(G)>4$ then box $(G) \leq 3$.

\section{Preliminaries}

Let $G$ be a simple, finite, undirected graph on $n$ vertices. The vertex set of $G$ is denoted as $V(G)$ and the edge set of $G$ is denoted as $E(G)$. Let $G^{\prime}$ be a graph such that $V\left(G^{\prime}\right)=V(G)$. Then, $G^{\prime}$ is a super graph of $G$ if $E(G) \subseteq E\left(G^{\prime}\right)$. We define the intersection of two graphs as follows: if $G_{1}$ and $G_{2}$ are two graphs such that $V\left(G_{1}\right)=$ $V\left(G_{2}\right)$, then the intersection of $G_{1}$ and $G_{2}$ denoted as $G=G_{1} \cap G_{2}$ is a graph with $V(G)=V\left(G_{1}\right)=V\left(G_{2}\right)$ and $E(G)=E\left(G_{1}\right) \cap E\left(G_{2}\right)$.

Let $G$ be a graph. Let $I_{1}, I_{2}, \ldots, I_{k}$ be $k$ interval graphs such that $G=I_{1} \cap I_{2} \cap$ $\cdots \cap I_{k}$. Then $I_{1}, I_{2}, \ldots, I_{k}$ is called an interval graph representation of $G$. The following equivalence is well known.

Lemma 2.1. (Roberts [7) The minimum $k$ such that there exists an interval graph representation of $G$ using $k$ interval graphs $I_{1}, I_{2}, \ldots, I_{k}$ is the same as box $(G)$.

Definition 2.1. Given a family $F$ of arcs on a circle, the overlap set of a point $P$ on the circle is the set of all arcs that contains the given point $P$ and is denoted by $\mathcal{O}(P)$. An overlap set with the smallest (respectively largest) number of arcs in it is called a minimum (respectively maximum) overlap set and its cardinality is denoted by $r_{\text {inf }}(F)$ (respectively $\left.r_{\text {sup }}(F)\right)$.

It is easy to see that the arcs in an overlap set induce a clique in the corresponding circular arc graph. 
Definition 2.2. For a circular arc graph $G, r_{i n f}(G)$ is defined as the minimum value of $r_{\text {inf }}(F)$ obtained over all possible family of arcs $F$ that can represent $G$.

Definition 2.3. A point $P$ is called a minimum overlap point if $|\mathcal{O}(P)|=r_{\text {inf }}(F)$. Note that there may be many minimum overlap points. Let $P_{\text {inf }}$ denote one of the minimum overlap points. All through the paper we shall use this special minimum overlap point as a reference point.

Definition 2.4. Each arc has two endpoints. The left endpoint $l(u)$ (respectively right endpoint $r(u)$ ) of arc $u$ is the first endpoint of $u$ encountered in an anticlockwise (respectively clockwise) traversal from any interior point of $u$. (The circle itself is not considered as an arc. A single point is also not considered as an arc. Thus the definition makes sense and every arc has a distinct left as well as a right end point. Note that these assumptions can be made without any loss of generality.)

Definition 2.5. An arc $v$ is said to be clockwise adjacent to an arc $u$ if $v$ belongs to the overlap set of $l(u)$. An arc $v$ is said to be anticlockwise adjacent to an arc $u$ if $v$ belongs to the overlap set of $r(u)$.

Definition 2.6. The start angle $s(u)$ (respectively end angle $e(u)$ ) of arc $u$ is the angle measured in clockwise direction from the line joining $l(u)$ (respectively $r(u)$ ) and center of the circle to the positive x-axis assuming that the center is at the origin.

Definition 2.7. Consider the set of end points $\{l(u), r(u): u \in V\}$. A clockwise ordering $\sigma$ of the endpoints is obtained, if we traverse the circle in the clockwise direction starting from the point $P_{\text {inf }}$ and list out the endpoints in the order in which they are seen, resolving any conflict arbitrarily. For a circular arc graph $G$ with a given circular arc representation, a clockwise ordering of the vertices can be obtained from the ordering of the left endpoints of their corresponding arcs in a similar way. In this paper we shall use $v_{i}$ to denote the ith vertex with respect to the clockwise ordering.

Definition 2.8. Let Int $(\boldsymbol{u})$ denote the set of half axes intersecting the arc $\boldsymbol{u}$. Then $|\operatorname{Int}(\boldsymbol{u})|$ is said to be the interception number of arc $\boldsymbol{u}$.

Let $G(\mathrm{~V}, \mathrm{E})$ be a circular arc graph with circular arc representation $\mathcal{C}_{0}$. From now on, the arc corresponding to vertex $\mathrm{u}$ will be denoted as $\mathbf{u}$. We assume $n \geq 2$ and $\Delta \geq 1$. Let us take a circle of unit radius centered at origin on the cartesian plane. Given a positive integer $\alpha \geq 2$, we define $\alpha$ reference axes as follows. The first reference axis $A_{0}$ is the same as the x-axis. For $1 \leq j<\alpha$ the $j$ th reference axis $A_{j}$ is the line passing through the origin and making an angle $\frac{\pi j}{\alpha}$ radian with the positive x-axis in anticlockwise direction. For the convenience of writing, we may sometimes use the notation $A_{j}$ even when $j \geq \alpha$ : In that case $A_{j}$ denotes the axis $A_{j} \bmod \alpha$. Also sometimes we use the notation $A_{-i}$ to denote $A_{\alpha-i}$. (We will refer to this set of reference axes as the system of reference axes based on $\alpha$ ).

Origin divides each reference axis into two halves. Each of them is called a half-axis. Clearly these $2 \alpha$ half-axes partition the interior of the circle into $2 \alpha$ regions - each region is called a sector. Note that each sector defines an arc on the circle. In this paper we will use the word sector to denote the arc defined by a sector also. An anticlockwise 
ordering of the half-axes is obtained, if we traverse the circle in anticlockwise direction starting from the positive $\mathrm{x}$-axis. We shall use the notation $H_{j}$ to denote the $j$ th half-axis in this ordering where $0 \leq j<2 \alpha$. It may be noted that the half axes obtained from the axis $A_{j}$ will get numbered $H_{j}$ and $H_{j+\alpha}$ according to the above mentioned numbering scheme. Sometimes we will refer $H_{j}$ as the positive half axis of $A_{j}$.

The sector in-between the half-axes $H_{j}$ and $H_{(j+1) \bmod 2 \alpha}$ is referred to as the $j$ th sector and is denoted as $S_{j}$ for $0 \leq j<2 \alpha$. (We sometimes use the notation $S_{-i}$ to denote $\left.S_{2 \alpha-i}\right) . \quad H_{j}$ and $H_{(j+1)} \bmod 2 \alpha$ are said to be the right and left half-axis of $S_{j}$ respectively. We also define $A_{j \bmod \alpha}$ and $A_{(j+1) \bmod \alpha}$ respectively to be the right and left reference axis of $S_{j}$. Note that $A_{j \bmod \alpha}$ corresponds to the half-axis $H_{j}$ and $A_{(j+1) \bmod \alpha}$ corresponds to the half-axis $H_{(j+1) \bmod 2 \alpha}$.

Lemma 2.2. There exists a circular arc representation $\mathcal{C}$ of $G$ which satisfies the following properties

1. All endpoints of arcs are distinct.

2. Left endpoints of the arcs are uniformly placed over the perimeter of the circle i.e. $\left|s\left(\boldsymbol{u}_{i+1}\right)-s\left(\boldsymbol{u}_{i}\right)\right|=\frac{2 \Pi}{n}$ radian where $0 \leq i<n-1$ (Recall that $s(\boldsymbol{u})$ is the start angle of $\boldsymbol{u})$.

\section{Endpoints of the arcs do not lie on any reference axis.}

Proof. We leave it to the reader to verify that given the circular arc representation $\mathcal{C}_{0}$ for $G$, there exists a circular arc representation $\mathcal{C}_{1}$ for $G$ such that all the endpoints of the arcs are at distinct points on the circle.

Let $\sigma$ be clockwise ordering of the endpoints of arcs in $\mathcal{C}_{1}$. Now given the circular arc representation $\mathcal{C}_{1}$ for $G$ and clockwise ordering $\sigma$ of the endpoints of the arcs, we shall show that there is another circular arc representation $\mathcal{C}_{2}$ for $\mathrm{G}$ such that $\mid s\left(\mathbf{u}_{i+1}\right)-$ $s\left(\mathbf{u}_{i}\right) \mid=\frac{2 \Pi}{n}$ radian for $0 \leq i<n-1$. Let $l_{0}, l_{1}, \ldots, l_{n-1}$ be the left endpoints of the arcs in the clockwise ordering $\sigma$. Place $l_{i}$ at the point on the circle defined by the polar coordinates $\left(1,-\frac{2 \Pi}{n} i\right)$ for $0 \leq i<n$. Let $e_{i_{1}}, e_{i_{2}}, \ldots, e_{i_{t}}$ be the right endpoints that are in-between $l_{i}$ and $l_{(i+1) \bmod n}$ with respect to $\sigma$. Place $e_{i_{1}}, e_{i_{2}}, \ldots e_{i_{t}}$ at distinct points on the circle in that order in clockwise direction on the arc in-between $l_{i}$ and $l_{(i+1) \bmod n}$. It is easy to verify that $\mathcal{C}_{2}$ will be a valid circular arc representation of $G$.

Now with respect to $\mathcal{C}_{2}$ if some endpoint lies on some reference axis we can rotate the circular arc system on the circle by $\epsilon$ radian where $\epsilon$ is a sufficiently small positive real number, to get a circular arc representation $\mathcal{C}$ satisfying the desired properties.

From now on we will use this circular arc representation $\mathcal{C}$ which satisfies the properties specified in Lemma 2.2. Let $\sigma$ be the clockwise ordering of the endpoints of arcs in $\mathcal{C}$.

\section{Boxicity when $\Delta<\left\lfloor n \frac{(\alpha-1)}{2 \alpha}\right\rfloor, \alpha \in \mathbb{N}, \alpha \geq 2$}

In this section we will be considering circular arc graphs of maximum degree strictly less than $\left\lfloor n \frac{(\alpha-1)}{2 \alpha}\right\rfloor, \alpha \in \mathbb{N}, \alpha \geq 2$. We will assume that we have a circular arc family $F$ that represents $G$ and obeys Lemma 2.2 
Observation 1: If $\Delta<\left\lfloor n \frac{(\alpha-1)}{2 \alpha}\right\rfloor$, then each arc $u \in F$ is such that

1. length $(\mathrm{u})<\Pi\left(\frac{\alpha-1}{\alpha}\right)$.

2. $|\operatorname{Int}(u)| \leq(\alpha-1)$.

Proof of 1: If an arc has length at least $\Pi\left(\frac{\alpha-1}{\alpha}\right)$, then it will intersect the left endpoints of at least $\left\lfloor\frac{n(\alpha-1)}{2 \alpha}\right\rfloor$ other arcs in view of Lemma 2.2. (Recall that we are considering a unit circle and therefore the distance between any pair of adjacent left endpoints is $\left.\frac{2 \Pi}{n}\right)$.

Proof of 2: Since length $(\mathrm{u})<\Pi\left(\frac{\alpha-1}{\alpha}\right)$, u can entirely contain at most $(\alpha-2)$ sectors. Therefore $|\operatorname{Int}(u)| \leq(\alpha-1)$.

All the definitions and Lemmas given in this section are applicable only for arcs of length $<\Pi\left(\frac{\alpha-1}{\alpha}\right)$.

Definition 3.1. For every arc $\boldsymbol{u}$ the extreme sectors are the sectors containing the endpoints of arc $\boldsymbol{u}$. One of the extreme sectors will be called the head sector and the other will be called the tail sector denoted by head $(\boldsymbol{u})$ and tail $(\boldsymbol{u})$ respectively. The sector head $(\boldsymbol{u})$ is defined to be the extreme sector that contains $l(\boldsymbol{u})$ and tail $(\boldsymbol{u})$ is defined to be the extreme sector that contains $r(\boldsymbol{u})$.

With respect to a reference axis $A_{j}$ we define the image of a point $P$ on the circle as the point $P^{\prime}$ on the circle such that the line defined by $P P^{\prime}$ is perpendicular to $A_{j}$. We denote $P^{\prime}$ as $I m_{j}(P)$. When $P$ is on $A_{j}$ we take $\operatorname{Im}_{j}(P)=P$. If $\mathrm{X}$ is a set of points on the circle, we will use $\operatorname{Im}_{j}(X)$ to denote the set $\left\{\operatorname{Im}_{j}(P): P \in X\right\}$.

Lemma 3.1. Let $X$ and $Y$ be the t-th sector starting from $H_{j}$ (i.e. the positive half axis of $\left.A_{j}\right)$ in the clockwise and anticlockwise direction respectively. Then $\operatorname{Im}_{j}(X)=Y$ and $\operatorname{Im}_{j}(Y)=X$.

Proof. Left to the reader.

Note that the $t$ th sector starting from $H_{j}$ for $0 \leq j<\alpha$ in the clockwise direction is $S_{(j-t) \bmod 2 \alpha}$ whereas the $t$ th sector starting from $H_{j}$ in the anticlockwise direction is $S_{(j+t-1) \bmod 2 \alpha}$.

Lemma 3.2. $\operatorname{Im}_{j}\left(S_{k}\right)=S_{h}$ where $h=(2 j-k-1) \bmod 2 \alpha$ for $0 \leq k<2 \alpha$ and $0 \leq j<\alpha$.

Proof. If $k<j, S_{k}$ is the $(j-k)$ th sector from $H_{j}$ in clockwise direction and therefore $\operatorname{Im}_{j}\left(S_{k}\right)$ will be the $(j-k)$ th sector from $H_{j}$ in the anticlockwise direction namely $S_{2 j-k-1}$. If $k \geq j, S_{k}$ is the $(k-j+1)$ th sector from $H_{j}$ in anticlockwise direction. So $\operatorname{Im}_{j}\left(S_{k}\right)=S_{(2 j-k-1) \bmod 2 \alpha}$ as required.

Lemma 3.3. For any reference axis $A_{j}$, for $0 \leq j<\alpha, \operatorname{Im}_{j}(l(\boldsymbol{u}))=r\left(\operatorname{Im}_{j}(\boldsymbol{u})\right)$ and $\operatorname{Im}_{j}(r(\boldsymbol{u}))=l\left(\operatorname{Im}_{j}(\boldsymbol{u})\right)$.

Proof. Left to the reader. 
Definition 3.2. With respect to a reference axis $A_{j}$ we define the projection of a point $P$ on the circle as the point $P^{\prime}$ on $A_{j}$ such that the line defined by $P P^{\prime}$ is perpendicular to $A_{j}$. We denote $P^{\prime}$ as $\operatorname{Proj}_{j}(P)$. When $P$ is on $A_{j}$ we take $\operatorname{Proj}_{j}(P)=P$. If $X$ is a set of points on the circle, we will use $\operatorname{Proj}_{j}(X)$ to denote the set $\left\{\operatorname{Proj}_{j}(P): P \in X\right\}$.

Note that the reference axis $A_{j}$ can be considered to be obtained by rotating the $\mathrm{x}$-axis (i.e. the real line) about the origin by an angle of $\frac{\pi j}{\alpha}$ radian in the anticlockwise direction. Then clearly there is a natural bijection between the points of $A_{j}$ and the set of real numbers namely the one which maps the positive x-axis to the positive half axis $H_{j}$ of $A_{j}$. For $\mathrm{X} \subset A_{j}$ let $X^{\prime} \subset \mathbb{R}$ be the set of real numbers that corresponds to $\mathrm{X}$ with respect to this bijection. Then we define $\inf X$ (respectively $\sup X)$ to be the point on $A_{j}$ that corresponds to $\inf X^{\prime}\left(\right.$ respectively $\left.\sup X^{\prime}\right)$.

Fact 3.4. If $P$ and $P^{\prime}$ are points on the circle such that $\operatorname{Proj}_{j}(P)=\operatorname{Proj}_{j}\left(P^{\prime}\right)$ for some $j$, where $0 \leq j<\alpha$. Then either $\operatorname{Im}_{j}(P)=P^{\prime}$ or $P=P^{\prime}$.

Proof. Left to the reader.

Lemma 3.5. Let $P, P^{\prime}$ be points on the circle such that $P \neq P^{\prime}, P \in S_{k}$ (where $0 \leq k<2 \alpha)$ and $\operatorname{Proj}_{j}(P)=\operatorname{Proj}_{j}\left(P^{\prime}\right)$ (where $\left.0 \leq j<\alpha\right)$. Then $P^{\prime} \in S_{h}$ where $h=(2 j-k-1) \bmod 2 \alpha$.

Proof. By fact 3.4, $S_{h}=\operatorname{Im}_{j}\left(S_{k}\right)$. Then $h=(2 j-k-1) \bmod 2 \alpha$ by Lemma 3.2 .

Definition 3.3. If Int $(\boldsymbol{u}) \neq \emptyset$ then let $H_{j_{1}}, H_{j_{2}}, \ldots, H_{j_{t}}$ be the set of half axes in Int $(\boldsymbol{u})$ in the order in which they appear, as one traverses the arc in the anticlockwise direction. (It may be noted that if the arc is properly contained in one sector then $\operatorname{Int}(\boldsymbol{u})=\emptyset$ ). Now median half axis (respectively median reference axis) with respect to arc $\boldsymbol{u}$

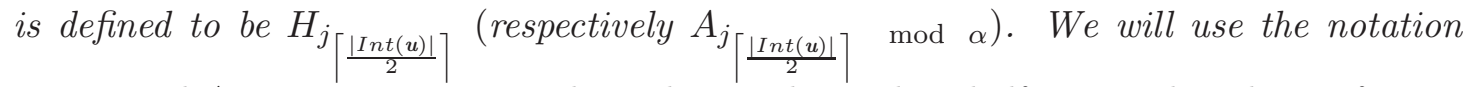
$H_{m(\boldsymbol{u})}$ and $A_{m(\boldsymbol{u}) \bmod \alpha}$ respectively to denote the median half axis and median reference axis with respect to arc $\boldsymbol{u}$.

The proof of our main theorem (Theorem 3.10) involves understanding the image of an arc $\mathbf{u}$ with respect to $A_{0}$ and $A_{1}$. It also turns out that we have to consider various cases based on $|\operatorname{Int}(\mathbf{u})|$. For a rigorous presentation of the proof we need to calculate the sector numbers of head $(\mathbf{u}), \operatorname{tail}(\mathbf{u})$ and their images with respect to $A_{0}$ and $A_{1}$. To avoid clumsiness within the proof, we have collected the required information in the Table 1.

Let $\mathbf{u}$ be an arc such that $|\operatorname{Int}(\mathbf{u})| \geq 1$. We assume that $m(\mathbf{u})=0$. Let $\mathrm{p}=\left\lceil\frac{|\operatorname{Int}(\mathbf{u})|}{2}\right\rceil$. The first column specifies the condition on $|\operatorname{Int}(\mathbf{u})|$ and the axis about which the image is taken. The next four columns specify the sector numbers corresponding to head $(\mathbf{u})$, $\operatorname{Im}_{j}(\operatorname{head}(\mathbf{u}))$, tail $(\mathbf{u})$ and $\operatorname{Im}_{j}(\operatorname{tail}(\mathbf{u}))$ respectively.

For the benefit of the reader we explain here how the entries in the first row of the Table are obtained:

When $|\operatorname{Int}(\mathbf{u})|$ is even clearly head $(\mathbf{u})$ is the $(p+1)$ th sector from $H_{m(\mathbf{u})}=H_{0}$ in anticlockwise direction, i.e. $S_{p}$. According to Lemma [3.2, $\operatorname{Im}_{0}(\operatorname{head}(\mathbf{u}))=S_{-(p+1)}$. 
Similarly tail $(\mathbf{u})$ is the $p$ th sector from $H_{0}$ in the clockwise direction that is $\operatorname{tail}(\mathbf{u})=S_{-p}$. According to Lemma $3.2 \operatorname{Im}_{0}(\operatorname{tail}(\mathbf{u}))=S_{p-1}$. The entries in the remaining rows are obtained in a similar way. (We use the notation $\operatorname{len}(\mathbf{u}), \operatorname{len}_{h}(\mathbf{u})$ and $\operatorname{len}_{t}(\mathbf{u})$ to denote length $(\mathbf{u})$, length $(\mathbf{u} \cap \operatorname{head}(\mathbf{u}))$ and length $(\mathbf{u} \cap \operatorname{tail}(\mathbf{u}))$ respectively. $)$

Table 1: Sector numbers corresponding to head(u), tail(u) and their images with respect to median axes for an arc $\mathbf{u}$ with $\mathrm{m}(\mathbf{u})=0$

\begin{tabular}{|c|c|c|c|c|}
\hline \multirow[t]{2}{*}{ Condition } & \multicolumn{4}{|c|}{ Sector Number $(\bmod 2 \alpha)$} \\
\hline & head $(\mathbf{u})$ & $\operatorname{Im}_{j}(\operatorname{head}(\mathbf{u}))$ & $\operatorname{tail}(\mathbf{u})$ & $\operatorname{Im}_{j}(\operatorname{tail}(\mathbf{u}))$ \\
\hline $\begin{array}{l}|\operatorname{Int}(\mathbf{u})| \text { is even } \\
\text { and } \mathbf{j}=0\end{array}$ & $S_{p}$ & $S_{-(p+1)}$ & $S_{-p}$ & $S_{p-1}$ \\
\hline $\begin{array}{l}|\operatorname{Int}(\mathbf{u})| \text { is even } \\
\text { and } \mathbf{j}=1\end{array}$ & $S_{p}$ & $S_{-p+1}$ & $S_{-p}$ & $S_{p+1}$ \\
\hline $\begin{array}{l}|\operatorname{Int}(\mathbf{u})| \text { is odd } \\
\text { and } \mathbf{j}=0\end{array}$ & $S_{p-1}$ & $S_{-p}$ & $S_{-p}$ & $S_{p-1}$ \\
\hline $\begin{array}{l}|\operatorname{Int}(\mathbf{u})| \text { is odd } \\
\text { and } \mathrm{j}=1\end{array}$ & $S_{p-1}$ & $S_{-p+2}$ & $S_{-p}$ & $S_{p+1}$ \\
\hline
\end{tabular}

Theorem 3.6. Let $\boldsymbol{u}$ and $\boldsymbol{v}$ be arcs such that $\boldsymbol{v} \cap \boldsymbol{u}=\emptyset$, $\operatorname{len}(\boldsymbol{u}) \leq \operatorname{len}(\boldsymbol{v})$. Let $|\operatorname{Int}(\boldsymbol{u})| \geq 2$ and therefore $p \geq 1$. Let $m(\boldsymbol{u})=0$.

1. If $|\operatorname{Int}(\boldsymbol{u})|$ is even

(a) If $\boldsymbol{v} \cap \operatorname{Im}_{0}(\boldsymbol{u}) \neq \emptyset$ then $\boldsymbol{v}$ must be anticlockwise adjacent to $\operatorname{Im}_{0}(\boldsymbol{u})$.

(b) If $\boldsymbol{v} \cap \operatorname{Im}_{1}(\boldsymbol{u}) \neq \emptyset$ then $\boldsymbol{v}$ must be clockwise adjacent to $\operatorname{Im}_{1}(\boldsymbol{u})$.

2. If $|\operatorname{Int}(\boldsymbol{u})|$ is odd and $\operatorname{len}_{h}(\boldsymbol{u})>\operatorname{len}_{t}(\boldsymbol{u})$

(a) If $\boldsymbol{v} \cap \operatorname{Im}_{0}(\boldsymbol{u}) \neq \emptyset$ then $\boldsymbol{v}$ must be anticlockwise adjacent to $\operatorname{Im}_{0}(\boldsymbol{u})$.

(b) If $\boldsymbol{v} \cap \operatorname{Im}_{1}(\boldsymbol{u}) \neq \emptyset$ then $\boldsymbol{v}$ must be clockwise adjacent to $\operatorname{Im}_{1}(\boldsymbol{u})$.

3. If $|\operatorname{Int}(\boldsymbol{u})|$ is odd and $\operatorname{len}_{h}(\boldsymbol{u})=\operatorname{len}_{t}(\boldsymbol{u})$ then $\boldsymbol{v} \cap \operatorname{Im}_{0}(\boldsymbol{u})=\emptyset$.

Proof of 1(a): Assume that $|\operatorname{Int}(\mathbf{u})|=2 p$. Since $\operatorname{len}(\mathbf{v}) \geq \operatorname{len}(\mathbf{u})=\operatorname{len}\left(\operatorname{Im}_{0}(\mathbf{u})\right)$, $\mathbf{v} \not \subset \operatorname{Im}_{0}(\mathbf{u})$. Now since $\mathbf{v} \cap \operatorname{Im}_{0}(\mathbf{u}) \neq \emptyset$ it follows that $\mathbf{v}$ contains either $l\left(\operatorname{Im}_{0}(\mathbf{u})\right)$ or $r\left(\operatorname{Im}_{0}(\mathbf{u})\right)$ i.e. $\mathbf{v}$ is either clockwise or anticlockwise adjacent to $\operatorname{Im}_{0}(\mathbf{u})$. According to Table $1, \operatorname{Im}_{0}(\operatorname{tail}(\mathbf{u}))=S_{p-1}$. Note from Table 1 that $\operatorname{tail}(\mathbf{u})=S_{-p}$ and $\operatorname{head}(\mathbf{u})=S_{p}$. Therefore $S_{p-1} \subset \mathbf{u}$ since $S_{p-1}$ is a sector strictly in-between $S_{p}$ and $S_{-p}$ in the clockwise direction, since $p \geq 1$. So recalling Lemma 3.3 we have $\left.l\left(\operatorname{Im}_{0}(\mathbf{u})\right)\right)=\operatorname{Im}_{0}(r(\mathbf{u})) \in \operatorname{Im}_{0}(\operatorname{tail}(\mathbf{u}))=S_{p-1} \subset \mathbf{u}$. Therefore $l\left(\operatorname{Im}_{0}(\mathbf{u})\right) \notin \mathbf{v}$ since $\mathbf{u} \cap \mathbf{v}=\emptyset$. It follows that $r\left(\operatorname{Im}_{0}(\mathbf{u})\right) \in \mathbf{v}$ i.e. $\mathbf{v}$ is anticlockwise adjacent to $\operatorname{Im}_{0}(\mathbf{u})$.

Proof of 1(b): The proof is similar to that of $1\left(\right.$ a). According to Table $1, \operatorname{Im}_{1}($ head $(\mathbf{u}))=$ $S_{h^{\prime}}$ where $h^{\prime}=(-p+1)$. Note that the sector $S_{h^{\prime}}$ is strictly in-between head $(\mathbf{u})=S_{p}$ and $\operatorname{tail}(\mathbf{u})=S_{-p}$ in the clockwise direction, since $p \geq 1$. So recalling Lemma 3.3 and using Table 1, we have $\left.r\left(\operatorname{Im}_{1}(\mathbf{u})\right)\right)=\operatorname{Im}_{1}(l(\mathbf{u})) \in \operatorname{Im}_{1}(h e a d(\mathbf{u}))=S_{h^{\prime}} \subset \mathbf{u}$. Therefore $r\left(\operatorname{Im}_{1}(\mathbf{u})\right) \notin \mathbf{v}$ since $\mathbf{u} \cap \mathbf{v}=\emptyset$. It follows that $l\left(\operatorname{Im}_{1}(\mathbf{u})\right) \in \mathbf{v}$ i.e. $\mathbf{v}$ is clockwise adjacent 
to $\operatorname{Im}_{1}(\mathbf{u})$.

Proof of 2(a): Let $|\operatorname{Int}(\mathbf{u})|=2 p+1$ and $\operatorname{len}_{h}(\mathbf{u})>\operatorname{len}_{t}(\mathbf{u})$. Then $l\left(\operatorname{Im}_{0}(\mathbf{u})\right)=$ $\operatorname{Im}_{0}(r(\mathbf{u})) \in \operatorname{Im}_{0}(\operatorname{tail}(\mathbf{u}))=S_{p-1}=\operatorname{head}(\mathbf{u})$. Since $\operatorname{len}_{h}(\mathbf{u})>\operatorname{len}_{t}(\mathbf{u})=\operatorname{len}\left(\operatorname{Im}_{0}(\mathbf{u} \cap\right.$ $\operatorname{tail}(\mathbf{u})))$. We infer that $l\left(\operatorname{Im}_{0}(\mathbf{u})\right) \in \mathbf{u}$ and therefore $l\left(\operatorname{Im}_{0}(\mathbf{u})\right) \notin \mathbf{v}$. Therefore $r\left(\operatorname{Im}_{0}(\mathbf{u})\right) \in \mathbf{v}$ i.e. $\mathbf{v}$ is anticlockwise adjacent to $\operatorname{Im}_{0}(\mathbf{u})$.

Proof of 2(b): The proof is similar to that of 1(a). According to Table 1, $\operatorname{Im}_{1}$ (head(u)) $=S_{h^{\prime}}$ where $h^{\prime}=(-p+2)$. Since $\operatorname{head}(\mathbf{u})=S_{p-1}$ and $\operatorname{tail}(\mathbf{u})=S_{-p}, S_{h^{\prime}}$ is strictly in-between head $(\mathbf{u})$ and $\operatorname{tail}(\mathbf{u})$ in the clockwise direction since $p \geq 2$. So recalling Lemma 3.3 we have $r\left(\operatorname{Im}_{1}(\mathbf{u})\right)=\operatorname{Im}_{1}(l(\mathbf{u})) \in \operatorname{Im}_{1}(\operatorname{head}(\mathbf{u}))=S_{h^{\prime}} \subset \mathbf{u}$. Therefore $r\left(\operatorname{Im}_{1}(\mathbf{u})\right) \notin \mathbf{v}$ since $\mathbf{u} \cap \mathbf{v}=\emptyset$. It follows that $l\left(\operatorname{Im}_{1}(\mathbf{u})\right) \in \mathbf{v}$ i.e. $\mathbf{v}$ is clockwise adjacent to $\operatorname{Im}_{1}(\mathbf{u})$.

Proof of 3: When $|\operatorname{Int}(\mathbf{u})|$ is odd then $\operatorname{Im}_{0}($ head $(\mathbf{u}))=S_{-p}=\operatorname{tail}(\mathbf{u})$ and $\operatorname{Im}_{0}(\operatorname{tail}(\mathbf{u}))$ $=S_{p-1}=\operatorname{head}(\mathbf{u})$. Now since $\operatorname{len}_{h}(\mathbf{u})=\operatorname{len}_{t}(\mathbf{u})$, we have $r(\mathbf{u})=\operatorname{Im}_{0}(l(\mathbf{u}))$ and $l(\mathbf{u})=\operatorname{Im}_{0}(r(\mathbf{u}))$. Hence $\operatorname{Im}_{0}(\mathbf{u})=\mathbf{u}$ and therefore $\mathbf{v} \cap \operatorname{Im}_{0}(\mathbf{u})=\emptyset$ since $\mathbf{u} \cap \mathbf{v}=\emptyset$.

Theorem 3.7. Let $\boldsymbol{u}$ and $\boldsymbol{v}$ be arcs such that $\boldsymbol{v} \cap \boldsymbol{u}=\emptyset$, len $(\boldsymbol{u}) \leq \operatorname{len}(\boldsymbol{v})$. Let $|\operatorname{Int}(\boldsymbol{u})|=1$ and therefore $p=1$. Let $m(\boldsymbol{u})=0$.

1. If $\operatorname{len}_{h}(\boldsymbol{u})>\operatorname{len}_{t}(\boldsymbol{u})$

(a) If $\boldsymbol{v} \cap \operatorname{Im}_{0}(\boldsymbol{u}) \neq \emptyset$ then $\boldsymbol{v}$ must be anticlockwise adjacent to $\operatorname{Im}_{0}(\boldsymbol{u})$.

(b) If $\boldsymbol{v} \cap \operatorname{Im}_{0}(\boldsymbol{u}) \neq \emptyset$ and $\boldsymbol{v} \cap \operatorname{Im}_{1}(\boldsymbol{u}) \neq \emptyset$ then $\boldsymbol{v}$ must be clockwise adjacent to $\operatorname{Im}_{1}(\boldsymbol{u})$.

2. If $\operatorname{len}_{h}(\boldsymbol{u})=\operatorname{len}_{t}(\boldsymbol{u})$ then $\boldsymbol{v} \cap \operatorname{Im}_{0}(\boldsymbol{u})=\emptyset$.

Proof of 1(a): When $\operatorname{len}_{h}(\mathbf{u})>\operatorname{len}_{t}(\mathbf{u})$ then $\left.l\left(\operatorname{Im}_{0}(\mathbf{u})\right)\right)=\operatorname{Im}_{0}(r(\mathbf{u})) \in \operatorname{Im}_{0}(\operatorname{tail}(\mathbf{u}))$ $=S_{0}=\operatorname{head}(\mathbf{u})$. Since $\operatorname{len}_{h}(\mathbf{u})>\operatorname{len}_{t}(\mathbf{u})=\operatorname{len}\left(\operatorname{Im}_{0}(\mathbf{u} \cap \operatorname{tail}(\mathbf{u}))\right)$. We infer that $l\left(\operatorname{Im}_{0}(\mathbf{u})\right) \in \mathbf{u}$ and therefore $l\left(\operatorname{Im}_{0}(\mathbf{u})\right) \notin \mathbf{v}$. Therefore $r\left(\operatorname{Im}_{0}(\mathbf{u})\right) \in \mathbf{v}$ i.e. $\mathbf{v}$ is anticlockwise adjacent to $\operatorname{Im}_{0}(\mathbf{u})$.

Proof of 1(b): According to Table 1, $\operatorname{Im}_{1}($ head $(\mathbf{u}))=S_{h^{\prime}}$ where $h^{\prime}=(-p+2)$ $=1$ and $\operatorname{Im}_{1}(\operatorname{tail}(\mathbf{u}))=S_{h^{\prime \prime}}$ where $h^{\prime \prime}=p+1=2$. So $\operatorname{Im}_{1}(\mathbf{u})$ goes from $S_{2}$ to $S_{1}$ in clockwise direction. Since $\operatorname{len}(\mathbf{v}) \geq \operatorname{len}(\mathbf{u})=\operatorname{len}\left(\operatorname{Im}_{1}(\mathbf{u})\right), \mathbf{v} \not \subset \operatorname{Im}_{1}(\mathbf{u})$. Therefore $\mathbf{v}$ is either clockwise or anticlockwise adjacent to $\operatorname{Im}_{1}(\mathbf{u})$. If possible let $l\left(\operatorname{Im}_{1}(\mathbf{u})\right) \notin \mathbf{v}$. But since $\mathbf{v} \cap \operatorname{Im}_{0}(\mathbf{u}) \neq \emptyset$, by case $1(\mathrm{a}), r\left(\operatorname{Im}_{0}(\mathbf{u})\right) \in \mathbf{v} \Longrightarrow \operatorname{Im}_{0}(l(\mathbf{u})) \in \mathbf{v} \Longrightarrow$ $\left(\mathbf{v} \cap \operatorname{Im}_{0}(\operatorname{head}(\mathbf{u}))\right) \neq \emptyset \Longrightarrow\left(\mathbf{v} \cap S_{-1}\right) \neq \emptyset$. It follows that $\mathbf{v}$ intersects all the sectors from $S_{1}$ to $S_{-1}$ in clockwise direction. (Note that this cannot be in anticlockwise direction since in that case $\left.l\left(\operatorname{Im}_{1}(\mathbf{u})\right) \in \mathbf{v}\right)$. Then $S_{0} \subset \mathbf{v}$. But it is not possible since $S_{0} \cap \mathbf{u} \neq \emptyset$ and $\mathbf{u} \cap \mathbf{v}=\emptyset$. Therefore $\mathbf{v}$ is clockwise adjacent to $\operatorname{Im}_{1}(\mathbf{u})$.

Proof of 2: When $\operatorname{len}_{h}(\mathbf{u})=\operatorname{len}(\mathbf{u})$ then $\operatorname{Im}_{0}(\operatorname{head}(\mathbf{u}))=S_{-1}=\operatorname{tail}(\mathbf{u})$ and $\operatorname{Im}_{0}(\operatorname{tail}(\mathbf{u}))$ $=S_{0}=\operatorname{head}(\mathbf{u})$. Now since $\operatorname{len}_{h}(\mathbf{u})=\operatorname{len}_{t}(\mathbf{u})$, we have $r(\mathbf{u})=\operatorname{Im}_{0}(l(\mathbf{u}))$ and $l(\mathbf{u})=\operatorname{Im}_{0}(r(\mathbf{u}))$. Hence $\operatorname{Im}_{0}(\mathbf{u})=\mathbf{u}$ and therefore $\mathbf{v} \cap \operatorname{Im}_{0}(\mathbf{u})=\emptyset$ since $\mathbf{u} \cap \mathbf{v}=\emptyset$. 


\subsection{Construction of interval graphs}

Let $G$ be a circular arc graph with maximum degree $\Delta<\left\lfloor\frac{n(\alpha-1)}{2 \alpha}\right\rfloor$. In this section we will construct $\alpha$ interval graphs $I_{0}, I_{1}, \ldots, I_{\alpha-1}$ such that $G=I_{0} \cap I_{1} \cap \ldots \cap I_{\alpha-1}$. Now consider a system of reference axes based on $\alpha$. To define $I_{j}$ we map each vertex $v \in V$ to an interval on $A_{j}$ by the mapping $\forall u \in V, g_{j}(u)=\left[\inf \operatorname{Proj}_{j}(\mathbf{u}), \sup \operatorname{Proj}_{j}(\mathbf{u})\right]$.

Lemma 3.8. For each interval graph $I_{j}, 0 \leq j<\alpha, E(G) \subseteq E\left(I_{j}\right)$.

Proof. Let $(u, v) \in E(G)$. There is a point $P \in \mathbf{u} \cap \mathbf{v}$. Clearly $\operatorname{Proj}_{j}(P) \in g_{j}(u) \cap g_{j}(v)$ for $0 \leq j<\alpha$. So, $E(G) \subseteq E\left(I_{j}\right)$ for $0 \leq j<\alpha$.

Lemma 3.9. If $(u, v) \notin E(G)$ then there exists some $j, 0 \leq j<\alpha$, such that $(u, v) \notin$ $E\left(I_{j}\right)$.

Proof. Since $(u, v) \notin E(G)$, we have $\mathbf{u} \cap \mathbf{v}=\emptyset$. Without loss of generality we can assume that $\operatorname{len}(\mathbf{u}) \leq \operatorname{len}(\mathbf{v})$. We consider the following cases

Case 1: $\operatorname{Int}(\mathbf{u})=\emptyset$. Then $\mathbf{u}$ is properly contained in some sector. Let $\mathbf{u} \subset S_{k} . A_{k_{l}}$ and $A_{k_{r}}$ be the left and right reference axes of $S_{k}$ where $k_{l}=(k+1) \bmod \alpha$ and $k_{r}=k \bmod \alpha$. In this case we will show that either $(u, v) \notin E\left(I_{k_{l}}\right)$ or $(u, v) \notin E\left(I_{k_{r}}\right)$. Suppose not. Then $g_{k_{l}}(u) \cap g_{k_{l}}(v) \neq \emptyset$ and therefore there exists some point $P_{u} \in \mathbf{u}$ and some point $P_{v} \in \mathbf{v}$ such that $\operatorname{Proj}_{k_{l}}\left(P_{u}\right)=\operatorname{Proj}_{k_{l}}\left(P_{v}\right)$. Then either $P_{u}=P_{v}$ or $\operatorname{Im}_{k_{l}}\left(P_{u}\right)=P_{v}$ by fact 3.4. But as $\mathbf{u} \cap \mathbf{v}=\emptyset$ we have $P_{u} \neq P_{v}$. Hence according to Lemma 3.5, $P_{v}$ must belong to the sector $(2((k+1) \bmod \alpha)-k-1) \bmod 2 \alpha=(k+1) \bmod 2 \alpha$. Similarly if $g_{k_{r}}(u) \cap g_{k_{r}}(v) \neq \emptyset$ then there exists some point $Q_{u} \in \mathbf{u}$ and some point $Q_{v}$ $\in \mathbf{v}$ such that $\operatorname{Proj}_{k_{r}}\left(Q_{u}\right)=\operatorname{Proj}_{k_{r}}\left(Q_{v}\right)$. For similar reasons as above $Q_{v}$ must belong to the sector $((k-1) \bmod 2 \alpha)$. Thus $\mathbf{v}$ intersects the sectors $S_{(k-1)} \bmod 2 \alpha$ as well as $S_{(k+1) \bmod 2 \alpha}$. But as $\mathbf{v}$ does not intersect $\mathbf{u}, \mathbf{v}$ cannot pass through $S_{k}$. It follows that it intersects all the other $(2 \alpha-1)$ sectors except $S_{k}$. In particular $\mathbf{v}$ properly contains all the $(2 \alpha-3)$ sectors other than $S_{(k-1) \bmod 2 \alpha}, S_{k}$ and $S_{(k+1) \bmod 2 \alpha}$ and therefore $\operatorname{len}(\mathbf{v}) \geq$ $(2 \alpha-3) \times$ sector length $=\frac{2 \Pi(2 \alpha-3)}{2 \alpha} \geq \frac{\Pi(\alpha-1)}{\alpha}$. Since $\alpha \geq 2$ this contradicts Observation 1 , part 1 .

Case 2: When $\operatorname{Int}(\mathbf{u}) \neq \emptyset$ and $|\operatorname{Int}(\mathbf{u})|$ is even. If $\alpha=2$ then $\operatorname{len}(\mathbf{u})<\frac{\Pi(\alpha-1)}{\alpha}=\frac{\Pi}{2}$ and therefore $\mathbf{u}$ cannot properly contain a sector, since a sector has length $\frac{I 1}{2}$ in this case. Then $|\operatorname{Int}(\mathbf{u})| \leq 1$ contradicts the assumption. Therefore we can assume that $\alpha \geq 3$. Let $w=m(\mathbf{u}) \bmod \alpha$ and $w^{\prime}=(m(\mathbf{u})+1) \bmod \alpha$, where $m(\mathbf{u})$ is the median axis number of $\mathbf{u}$. Our intention is to show that either $(u, v) \notin E\left(I_{w}\right)$ or $(u, v) \notin E\left(I_{w^{\prime}}\right)$. Now for the ease of presentation we will renumber our system of reference axes such that $m(\mathbf{u})=0$. Note that it only corresponds to a rotation of the circular arc system in such a way that $H_{m(\mathbf{u})}$ comes to $H_{0}$. After this transformation the interval graphs $I_{w}$ and $I_{w^{\prime}}$ will also get renamed to $I_{0}$ and $I_{1}$ respectively. So now we have only to prove that either $(u, v) \notin E\left(I_{0}\right)$ or $(u, v) \notin E\left(I_{1}\right)$. Let $|\operatorname{Int}(\mathbf{u})|=2 p$. Let $P^{+}$and $P^{-}$be the intersection point of the circle with $H_{0}$ and $H_{\alpha}$ (i.e. positive and negative x-axis) respectively. If $g_{0}(u)$ $\cap g_{0}(v) \neq \emptyset$ then $\mathbf{v} \cap \operatorname{Im}_{0}(\mathbf{u}) \neq \emptyset$. Since $\mathbf{v} \cap \mathbf{u}=\emptyset$ according to Theorem 3.6 part 1 (a), $\mathbf{v}$ must be anticlockwise adjacent to $\operatorname{Im}_{0}(\mathbf{u})$. Therefore $r\left(\operatorname{Im}_{0}(\mathbf{u})\right) \in \mathbf{v}$ which implies that $\operatorname{Im}_{0}(l(\mathbf{u})) \in \mathbf{v}$ by Lemma 3.3. Similarly since $g_{1}(u) \cap g_{1}(v) \neq \emptyset$, according to Theorem 
3.6 part 1(b), v must be clockwise adjacent to $\operatorname{Im}_{1}(\mathbf{u})$. Therefore $l\left(\operatorname{Im}_{1}(\mathbf{u})\right) \in \mathbf{v}$ which implies that $\operatorname{Im}_{1}(r(\mathbf{u})) \in \mathbf{v}$ by Lemma 3.3. But according to Table 1, $\operatorname{Im}_{1}(r(\mathbf{u})) \in I m_{1}$ $(\operatorname{tail}(\mathbf{u}))=S_{p+1}$ and $\operatorname{Im}_{0}(l(\mathbf{u})) \in \operatorname{Im}_{0}(\operatorname{head}(\mathbf{u}))=S_{-(p+1)}$. Therefore $\mathbf{v}$ intersects the sectors $S_{-(p+1)}$ as well as $S_{p+1}$. Now since $(p+1) \leq(\alpha-1)$ for $\alpha \geq 3$ (see Observation 1, part 1), the sector $S_{p+1}$ belongs to the half circle above $A_{0}$ (i.e. x-axis). Similarly the sector $S_{-(p+1)}$ belongs to the half circle below $A_{0}$ (i.e. x-axis). It follows that the arc $\mathbf{v}$ should intersect $A_{0}$ either at $P^{+}$or $P^{-}$. But in view of definition of median axis $P^{+} \in \mathbf{u}$. Since $\mathbf{u} \cap \mathbf{v}=\emptyset$ we have $P^{-} \in \mathbf{v}$.

So we infer that $\mathbf{v}$ intersects all the sectors from $S_{-(p+1)}$ to $S_{p+1}$ in the clockwise

direction.

Moreover we can infer that $\mathbf{v}$ properly contains all the sectors strictly in-between $S_{-(p+1)}$ and $S_{p+1}$ in the clockwise direction (if there is any such sector). Note that since $\operatorname{head}(\mathbf{u})=S_{p}$ and $\operatorname{tail}(\mathbf{u})=S_{-p}$, u properly contains all the sectors strictly in-between $S_{p}$ and $S_{-p}$ in the clockwise direction (if there is any such sector).

Thus $\mathbf{v} \cup \mathbf{u}$ properly contains all the $(2 \alpha-4)$ sectors other than $S_{p}, S_{-p}, S_{-(p+1)}$ and $S_{p+1}$ namely head(u), tail $(\mathbf{u}), \operatorname{Im}_{0}($ head $(\mathbf{u}))$ and $\operatorname{Im}_{1}(\operatorname{tail}(\mathbf{u}))$.

Claim 1: If $|\operatorname{Int}(\mathbf{u})|$ is even

1. $\left(\operatorname{len}_{h}(\mathbf{u})+\operatorname{len}\left(\mathbf{v} \cap S_{-(p+1)}\right)\right) \geq \operatorname{len}\left(S_{-(p+1)}\right)$.

2. $\left(\operatorname{len}_{t}(\mathbf{u})+\operatorname{len}\left(\mathbf{v} \cap S_{p+1}\right)\right) \geq \operatorname{len}\left(S_{p+1}\right)$.

Proof. Since $\operatorname{len}_{h}(\mathbf{u})=\operatorname{len}(\mathbf{u} \cap \operatorname{head}(\mathbf{u}))=\operatorname{len}\left(\operatorname{Im}_{0}(\mathbf{u}) \cap \operatorname{Im}_{0}(\right.$ head $\left.(\mathbf{u}))\right)$ and recalling from Table 1 that $\operatorname{Im}_{0}($ head $(\mathbf{u}))=S_{-(p+1)}$ it is sufficient to show that $\operatorname{len}\left(\operatorname{Im}_{0}(\mathbf{u}) \cap\right.$ $\left.S_{-(p+1)}\right)+\operatorname{len}\left(\mathbf{v} \cap S_{-(p+1)}\right) \geq \operatorname{len}\left(S_{-(p+1)}\right)$. Let $P=\operatorname{Im}_{0}(l(\mathbf{u}))=r\left(\operatorname{Im}_{0}(\mathbf{u})\right)$. We know that $P \in \operatorname{Im}_{0}(\operatorname{head}(\mathbf{u}))=S_{-(p+1)}$. Let $P_{L}=l\left(S_{-(p+1)}\right)$ and $P_{R}=r\left(S_{-(p+1)}\right)$. Note that $\operatorname{head}(\mathbf{u}) \cap \mathbf{u}$ is an arc from $l(\mathbf{u})$ to $r($ head $(\mathbf{u}))$ in the clockwise direction. Therefore $\operatorname{Im}_{0}(\mathbf{u} \cap \operatorname{head}(\mathbf{u}))=\operatorname{Im}_{0}(\mathbf{u}) \cap \operatorname{Im}_{0}(\operatorname{head}(\mathbf{u}))=\left(\operatorname{Im}_{0}(\mathbf{u}) \cap S_{-(p+1)}\right)$ is the arc from $\operatorname{Im}_{0}(l(\mathbf{u}))=r\left(\operatorname{Im}_{0}(\mathbf{u})\right)=P$ to $\operatorname{Im}_{0}(r(\operatorname{head}(\mathbf{u})))=l\left(\operatorname{Im}_{0}(\operatorname{head}(\mathbf{u}))\right)=P_{L}$ within the sector $\operatorname{Im}_{0}(\operatorname{head}(\mathbf{u}))=S_{-(p+1)}$. Since $g_{0}(u) \cap g_{0}(v) \neq \emptyset$ by Theorem [3.6 part $1(\mathrm{a}), r\left(\operatorname{Im}_{0}(\mathbf{u})\right)=P \in \mathbf{v}$. Moreover by statement (1), $\mathbf{v}$ intersects all the sectors from $S_{-(p+1)}$ to $S_{p+1}$ in the clockwise direction. It follows that $P_{R} \in \mathbf{v}$. In other words the arc from $\mathrm{P}$ to $P_{R}$ (in the clockwise direction) is contained in $\mathbf{v}$. Thus we have $\left(\operatorname{len}_{h}(\mathbf{u})+\operatorname{len}\left(\mathbf{v} \cap S_{-(p+1)}\right)\right) \geq \operatorname{len}\left(S_{-(p+1)}\right)$.

Proof of part 2 is similar as that of the part 1 except that instead of head(u) we have to use tail( $\mathbf{u})$. Moreover instead of reference axis $A_{0}$ we have to use $A_{1}$ and sector $S_{p+1}$ should replace $S_{-(p+1)}$.

Thus in view of statement (21) and claim 1 and recalling that the length of one sector is $\frac{\Pi}{\alpha}$ we can say that $\operatorname{len}(\mathbf{u})+\operatorname{len}(\mathbf{v}) \geq(2 \alpha-2) \frac{\Pi}{\alpha}$. Now since $\operatorname{len}(\mathbf{v}) \geq \operatorname{len}(\mathbf{u})$ we get $\operatorname{len}(\mathbf{v}) \geq(\alpha-1) \frac{\Pi}{\alpha}$, a contradiction to Observation 1 , part 1 .

Case 3: If $|\operatorname{Int}(\boldsymbol{u})| \geq 1$ and $|\operatorname{Int}(\boldsymbol{u})|$ is odd. As we did in case 2, we first renumber 
our system of reference axes such that $m(\mathbf{u})=0$, i.e. we rotate the circular arc system in such a way that $H_{m(\mathbf{u})}$ comes to $H_{0}$. Note that after the rotation $H_{m(\mathbf{u})+1}$ will correspond to $H_{1}$ and $H_{m(\mathbf{u})-1}$ will correspond to $H_{-1}$. We consider the following subcases.

Subcase 3.1: $\operatorname{len}_{h}(\boldsymbol{u})=\operatorname{len}_{t}(\boldsymbol{u})$. By Theorem 3.6 part $3, \operatorname{Im}_{0}(\mathbf{u})=\mathbf{u}$ and hence $g_{0}(u) \cap g_{0}(v)=\emptyset$. Therefore we have $(u, v) \notin E\left(I_{0}\right)$ as required.

Subcase 3.2: $\operatorname{len}_{h}(\boldsymbol{u}) \neq l e n_{t}(\boldsymbol{u})$. Our proof requires that we consider two cases namely

1. $\operatorname{len}_{h}(\mathbf{u})>\operatorname{len}(\mathbf{u})$

2. $\operatorname{len}_{h}(\mathbf{u})<\operatorname{len}_{t}(\mathbf{u})$

In the first case our intention is to show that either $(u, v) \notin E\left(I_{m(\mathbf{u}) \bmod \alpha}\right)$ or $(u, v) \notin$ $E\left(I_{(m(\mathbf{u})+1) \bmod \alpha}\right)$ and in the second case our intention is to show that either $(u, v) \notin$ $E\left(I_{m(\mathbf{u}) \bmod \alpha}\right)$ or $(u, v) \notin E\left(I_{(m(\mathbf{u})-1) \bmod \alpha}\right)$. In fact there is no need to analyze these

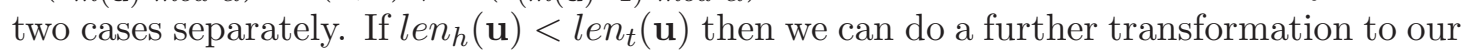
circular arc system namely a reflection about the x-axis. Note that after the reflection the left and right endpoints of each arc gets interchanged (see Lemma 3.3). Therefore in this transformed circular arc system $\operatorname{len}_{h}(\mathbf{u})>\operatorname{len}_{t}(\mathbf{u})$. Also note that the numbering of the reference axes has also changed due to reflection: $A_{0}$ still corresponds to $A_{0}$ but $A_{1}$ corresponds to $A_{-1}$ and $A_{-1}$ corresponds to $A_{1}$. Thus the half axis that was originally numbered $m(\mathbf{u})$ will be numbered 0 and $(m(\mathbf{u})-1)$ will be numbered 1 after the rotation and reflection. Note that the interval graphs are also renamed after this transformation. Thus in both cases we need only to prove that either $(u, v) \notin E\left(I_{0}\right)$ or $(u, v) \notin E\left(I_{1}\right)$. Suppose not. Then we claim the following

Claim 2: $\mathbf{v} \cup \mathbf{u}$ properly contains all the $(2 \alpha-4)$ sectors other than head $(\mathbf{u})$, tail $(\mathbf{u})$, $S_{p}$ and $S_{p+1}$.

Proof. If $\alpha=2,(2 \alpha-4)=0$ and the claim is trivially true. Thus we can assume $\alpha \geq 3$. Let $P^{+}$and $P^{-}$be the intersection point of the circle with $H_{0}$ and $H_{\alpha}$ (i.e. positive and negative X-axis) respectively. If $g_{0}(u) \cap g_{0}(v) \neq \emptyset$ then $\mathbf{v} \cap \operatorname{Im}_{0}(\mathbf{u}) \neq \emptyset$. Since $\mathbf{v} \cap \mathbf{u}=\emptyset$, using Theorem 3.6 part 2(a) and Theorem 3.7 part 1(a), we can conclude that $\mathbf{v}$ must be anticlockwise adjacent to $\operatorname{Im}_{0}(\mathbf{u})$. So according to Lemma 3.3, $\operatorname{Im}_{0}(l(\mathbf{u})) \in \mathbf{v}$. Similarly since $g_{1}(u) \cap g_{1}(v) \neq \emptyset$, by Theorem 3.6 part 2(b) and Theorem 3.7 part 1(b) $\mathbf{v}$ must be clockwise adjacent to $\operatorname{Im}_{1}(\mathbf{u})$. Hence according to Lemma $3.3 \operatorname{Im}_{1}(r(\mathbf{u})) \in \mathbf{v}$. Thus according to Table $1, \mathbf{v}$ intersects the sectors $S_{-p}$ as well as $S_{p+1}$. Now since $(p+1) \leq(\alpha-1)$ for $\alpha \geq 3$ (see Observation 1 part 2), the sector $S_{p+1}$ belongs to the half circle above $A_{0}$ (i.e. x-axis). Similarly the sector $S_{-p}$ belongs to the half circle below $A_{0}$ (i.e. x-axis). It follows that the arc $\mathbf{v}$ should intersect $A_{0}$ either at $P^{+}$or $P^{-}$. But in view of definition of median axis $P^{+} \in \mathbf{u}$. Since $\mathbf{u} \cap \mathbf{v}=\emptyset$ we have $P^{-} \in \mathbf{v}$. So we infer the following

$$
\text { If } \alpha \geq 3 \text { then } \mathbf{v} \text { intersects all the sectors from } S_{-p} \text { to } S_{p+1} \text { in the clockwise }
$$

direction.

Moreover we can infer that $\mathbf{v}$ properly contains all the sectors strictly in-between $S_{-p}$ and $S_{p+1}$ in clockwise direction (if there is any such sector). Also note that since head $(\mathbf{u})=$ 
$S_{p-1}$ and $\operatorname{tail}(\mathbf{u})=S_{-p}, \mathbf{u}$ properly contains all the sectors strictly in-between $S_{p-1}$ to $S_{-p}$ in the clockwise direction (if there is any such sector). Therefore $\mathbf{v} \cup \mathbf{u}$ properly contains all the $(2 \alpha-4)$ sectors other than $S_{p-1}, S_{-p}, S_{p}$ and $S_{p+1}$ namely head(u), $\operatorname{tail}(\mathbf{u}), S_{p}$ and $S_{p+1}$.

Observation 2: Note that statement (3) is valid even when $\alpha=2$. When $\alpha=2$ and $|\operatorname{Int}(\mathbf{u})|=1, p=1$. Since $g_{0}(u) \cap g_{0}(v) \neq \emptyset$ and $r\left(\operatorname{Im}_{0}(\mathbf{u})\right) \in S_{-1}, \mathbf{v} \cap S_{-1} \neq \emptyset$. Similarly since $g_{1}(u) \cap g_{1}(v) \neq \emptyset$ and $l\left(\operatorname{Im}_{1}(\mathbf{u})\right) \in S_{2}, \mathbf{v} \cap S_{2} \neq \emptyset$. Therefore $\mathbf{v}$ goes from $S_{-1}$ to $S_{2}$ in clockwise direction because otherwise $S_{0} \subset \mathbf{v}$, a contradiction to our assumption that $\mathbf{u} \cap \mathbf{v}=\emptyset$.

Claim 3: If $|\operatorname{Int}(\mathbf{u})|$ is odd and $\operatorname{len}_{h}(\mathbf{u})>\operatorname{len}_{t}(\mathbf{u})$

1. $\left(\operatorname{len}_{h}(\mathbf{u})+\operatorname{len}\left(\mathbf{v} \cap S_{-p}\right)\right) \geq \operatorname{len}\left(S_{-p}\right)$.

2. $\left(\operatorname{len}_{t}(\mathbf{u})+\operatorname{len}\left(\mathbf{v} \cap S_{p+1}\right)\right) \geq \operatorname{len}\left(S_{p+1}\right)$.

Proof. Since $\operatorname{len}_{h}(\mathbf{u})=\operatorname{len}(\mathbf{u} \cap \operatorname{head}(\mathbf{u}))=\operatorname{len}\left(\operatorname{Im}_{0}(\mathbf{u}) \cap \operatorname{Im}_{0}(\operatorname{head}(\mathbf{u}))\right)$ and recalling from Table 1 that $\operatorname{Im}_{0}(\operatorname{head}(\mathbf{u}))=S_{-p}$ it is sufficient to show that $\operatorname{len}\left(\operatorname{Im}_{0}(\mathbf{u}) \cap S_{-p}\right)$ $+\operatorname{len}\left(\mathbf{v} \cap S_{-p}\right) \geq \operatorname{len}\left(S_{-p}\right)$. Let $P=\operatorname{Im}_{0}(l(\mathbf{u}))=r\left(\operatorname{Im}_{0}(\mathbf{u})\right)$. We know that $P \in$ $\operatorname{Im}_{0}(\operatorname{head}(\mathbf{u}))=S_{-p}$. Let $P_{L}=l\left(S_{-p}\right)$ and $P_{R}=r\left(S_{-p}\right)$. Note that head $(\mathbf{u}) \cap \mathbf{u}$ is an arc from $1(\mathbf{u})$ to $\mathrm{r}($ head $(\mathbf{u}))$ in the clockwise direction. Therefore $\operatorname{Im}_{0}(\mathbf{u} \cap$ head $(\mathbf{u}))$ $=\operatorname{Im}_{0}(\mathbf{u}) \cap \operatorname{Im}_{0}(\operatorname{head}(\mathbf{u}))=\left(\operatorname{Im}_{0}(\mathbf{u}) \cap S_{-p}\right)$ is the $\operatorname{arc}$ from $\operatorname{Im}_{0}(l(\mathbf{u}))=r\left(\operatorname{Im}_{0}(\mathbf{u})\right)=P$ to $\operatorname{Im}_{0}(r(\operatorname{head}(\mathbf{u})))=l\left(\operatorname{Im}_{0}(\operatorname{head}(\mathbf{u}))\right)=P_{L}$ within the sector $\operatorname{Im}_{0}($ head $(\mathbf{u}))=S_{-p}$. Since $g_{0}(u) \cap g_{0}(v) \neq \emptyset$ by Theorem 3.6 part 2(a) and Theorem 3.7 part $1(\mathrm{a}), r\left(\operatorname{Im}_{0}(\mathbf{u})\right)$ $=P \in \mathbf{v}$. Moreover by statement (3) and Observation 2, $\mathbf{v}$ intersects all the sectors from $S_{-p}$ to $S_{p+1}$ in the clockwise direction. It follows that $P_{R} \in \mathbf{v}$. In other words the arc from $\mathrm{P}$ to $P_{R}$ (in the clockwise direction) is contained in $\mathbf{v}$. Thus we have $\left(\operatorname{len}_{h}(\mathbf{u})+\operatorname{len}\left(\mathbf{v} \cap S_{-p}\right)\right) \geq \operatorname{len}\left(S_{-p}\right)$.

Proof of part(2) is the same as that of the part(1) except that instead of head(u) we have to use tail $(\mathbf{u})$. Moreover instead of reference axis $A_{0}$ we have to use $A_{1}$ and sector $S_{p+1}$ should replace $S_{-p}$.

Now in view of claim 2, claim 3 and recalling that the length of one sector is $\frac{\Pi}{\alpha}$ we can say that $\operatorname{len}(\mathbf{u})+\operatorname{len}(\mathbf{v}) \geq(2 \alpha-2) \frac{\Pi}{\alpha}$. Now since $\operatorname{len}(\mathbf{v}) \geq \operatorname{len}(\mathbf{u})$ we get $\operatorname{len}(\mathbf{v}) \geq(\alpha-1) \frac{\Pi}{\alpha}$, a contradiction to Observation 1, part 1.

Combining Lemma 3.8 and 3.9 we have the following Theorem.

Theorem 3.10. For a circular arc graph $G$, box $(G) \leq \alpha$ when $\Delta<\left\lfloor\frac{n(\alpha-1)}{2 \alpha}\right\rfloor$.

\subsection{Tightness Result}

It would have been nice if we could show a circular arc graph $G$ with $\Delta=\frac{n(\alpha-1)}{2 \alpha}$ and $\operatorname{box}(G)>\alpha$ in order to demonstrate the tightness of the bound given in Theorem 3.10 , Unfortunately we are unable to construct such a graph. In this section we will show a circular arc graph $G$ with $\operatorname{box}(G)>\alpha$ and $\Delta=n \frac{(\alpha-1)}{2 \alpha}+\frac{n}{2 \alpha(\alpha+1)}+(\alpha+2)$. Thus the 
upper bound on $\Delta$ cannot be raised by more than an additive factor of $\frac{n}{2 \alpha(\alpha+1)}+(\alpha+2)$ $\approx \frac{n}{2 \alpha^{2}}$ keeping $\operatorname{box}(G) \leq \alpha$. We describe the graph $G$ below.

Let $V(G)$ be the disjoint union of 3 sets of vertices namely $S_{1}, S_{2}$ and $S_{3}$ such that $\left|S_{1}\right|=2 \alpha+2,\left|S_{2}\right|=2 \alpha+2$ and $\left|S_{3}\right|=n-(4 \alpha+4)$. (We will assume that $\mathrm{n}$ is divisible by $2 \alpha+2)$. Let $S_{1}=\left\{u_{0}, u_{1}, \ldots, u_{2 \alpha+1}\right\}, S_{2}=\left\{v_{0}, v_{1}, \ldots, v_{2 \alpha+1}\right\}$. Let $S_{3}$ contain the remaining $n-(4 \alpha+4)$ vertices. We will partition the set $S_{3}$ further into $2 \alpha+2$ subsets $H_{0}, H_{1}, \ldots, H_{2 \alpha+1}$ where $\left|H_{i}\right|=\frac{n}{2 \alpha+2}-2$ i.e. $S_{3}=H_{0} \cup H_{1} \cup \ldots \cup H_{2 \alpha+1}$. The circular arc graph $G$ on the vertex set $V(G)=S_{1} \cup S_{2} \cup S_{3}$ is constructed as follows.

Let us take a circle. Let $\mathrm{t}=2 \alpha+2$. Place $\mathrm{t}$ points on the circle such that the distance between any adjacent pair of points is equal. Let these points be $p_{0}, p_{1}, \ldots, p_{t-1}$ in clockwise direction. For each $\mathrm{i}, 0 \leq i \leq(2 \alpha+1), u_{i}$ is mapped to the circular arc from $p_{i}$ to $p_{(i+\alpha) \bmod t}$ in the clockwise direction. Similarly for each $\mathrm{i}, 0 \leq i \leq(2 \alpha+1), v_{i}$ is mapped to the circular arc from $p_{i}$ to $p_{(i+1)} \bmod t$ in the clockwise direction. For each i, $0 \leq i \leq(2 \alpha+1)$, the vertices in $H_{i}$ are mapped to arcs of sufficiently small length placed strictly in-between $p_{i}$ and $p_{(i+1)} \bmod t$ (i.e. strictly inside the arc corresponding to $v_{i}$ ) such that arcs in $H_{i}$ are pairwise non-intersecting.

Claim 1: Maximum degree of $G$ is $\frac{n \alpha}{2(\alpha+1)}+(\alpha+2)=\frac{n(\alpha-1)}{2 \alpha}+\frac{n}{2 \alpha(\alpha+1)}+(\alpha+2)$.

Proof. Any vertex in set $S_{1}$ is connected to $2 \alpha$ vertices in set $S_{1},(\alpha+2)$ vertices in set $S_{2}$ and $\alpha\left(\frac{n}{2(\alpha+1)}-2\right)$ vertices in set $S_{3}$. So maximum degree of any vertex in $S_{1}$ is $\frac{n \alpha}{2(\alpha+1)}+(\alpha+2)$.

Any vertex in set $S_{2}$ is connected to $(\alpha+2)$ vertices in set $S_{1}, 2$ vertices in set $S_{2}$ and $\left(\frac{n}{2(\alpha+1)}-2\right)$ vertices in set $S_{3}$. So maximum degree of any vertex in $S_{2}$ is $\frac{n}{2(\alpha+1)}+\alpha+2$. Any vertex in set $S_{3}$ is connected to $(\alpha-1)$ vertices in set $S_{1}$ and 1 vertex in set $S_{2}$. So maximum degree of any vertex in $S_{3}$ is $\alpha$.

So maximum degree of $G$ is $\frac{n \alpha}{2(\alpha+1)}+(\alpha+2)$.

Claim 2: $\operatorname{box}(G)>\alpha$.

Proof. It is easy to see that in $G, S_{1}$ induces a subgraph isomorphic to $\overline{(\alpha+1) K_{2}}$. (See the circular arc construction of $\overline{\left(\frac{n}{2}\right) K_{2}}$ given in the introduction). From [7], box $\left(\overline{\left.(\alpha+1) K_{2}\right)}\right.$ $=\alpha+1$ and therefore $\operatorname{box}(\mathrm{G})>\alpha$.

So there exists a graph on $\mathrm{n}$ vertices with maximum degree $\frac{n \alpha}{2(\alpha+1)}+(\alpha+2)$ and boxicity $>\alpha$.

\section{An upper bound based on minimum overlap set}

Cardinality of minimum overlap set is a parameter (see definition 2.3) one encounters frequently in the study of circular arc graphs. For example let $F$ be the family of arcs in a circular arc graph $G$ then $\chi(F) \leq r_{\text {sup }}(F)+r_{\text {inf }}(F)$ [2]. In the following theorem we relate $\operatorname{box}(G)$ with $r_{i n f}(G)$.

Theorem 4.1. For a circular arc graph $G$, box $(G) \leq r_{\text {inf }}(G)+1$ where $r_{\text {inf }}(G)$ is the cardinality of minimum overlap set with respect to any circular arc representation of $G$. 
From now on we will use $r_{\text {inf }}$ instead of $r_{\text {inf }}(G)$. Let $\mathcal{O}\left(P_{\text {inf }}\right)=\left\{\mathbf{w}_{1}, \mathbf{w}_{2}, \ldots, \mathbf{w}_{r_{\text {inf }}}\right\}$. We construct $r_{i n f}+1$ interval super graphs of $G$ say $I_{1}, I_{2}, \ldots, I_{r_{i n f}+1}$ as follows.

Construction of $I_{i}$ for $1 \leq i \leq r_{i n f}$ : For each $\mathbf{w}_{i}$ we will construct an interval graph $I_{i}$. To construct $I_{i}$ we map each vertex $v \in V(G)$ to an interval on the real line by the mapping

$$
\begin{aligned}
g_{i}(v) & =[0,1] & & \text { if } v=w_{i} \\
& =[1,2] & & \text { if } v \in N_{G}\left(w_{i}\right) . \\
& =[2,3] & & \text { if } v \in V(G)-\left(N_{G}\left(w_{i}\right) \cup\left\{w_{i}\right\}\right) .
\end{aligned}
$$

Construction of $I_{r_{i n f}+1}$ : Recall that according to definition 2.7, $\sigma$ is a clockwise ordering starting from $P_{\text {inf }}$. So $\sigma(l(\mathbf{v}))<\sigma(r(\mathbf{v}))$ when $\mathbf{v} \notin \mathcal{O}\left(P_{\text {inf }}\right)$. We define the mapping as follows:

$$
\begin{aligned}
g_{r_{\text {inf }}+1}(v) & =[1,2 n] \quad \text { if } \mathbf{v} \in \mathcal{O}\left(P_{\text {inf }}\right) \\
& =[\sigma(l(\mathbf{v})), \sigma(r(\mathbf{v}))] \quad \text { otherwise. }
\end{aligned}
$$

Lemma 4.2. For each interval graph $I_{j}$ where $1 \leq j \leq r_{\text {inf }}+1, E(G) \subseteq E\left(I_{j}\right)$.

Proof. We consider the following two cases

Case 1: When $1 \leq j \leq r_{\text {inf }}$. It is easy to see that for all $x \in N_{G}\left(w_{i}\right) \cup\left\{w_{i}\right\}, 1 \in g_{i}(x)$. So $N_{G}\left(w_{i}\right) \cup\left\{w_{i}\right\}$ induces a clique in $I_{i}$. Also for all $x \in V(G)-\left\{w_{i}\right\}, 2 \in g_{i}(x)$. So $V(G)-\left\{w_{i}\right\}$ induces a clique in $I_{i}$. Therefore we infer that $E(G) \subseteq E\left(I_{i}\right)$ for each $i$ $1 \leq i \leq r_{\text {inf }}$.

Case 2: When $j=r_{\text {inf }}+1$. For any edge $(u, v) \in E(G)$ we consider the following two cases

Subcase 2.1: $\mathbf{u} \in \mathcal{O}\left(P_{\text {inf }}\right)$ or $\mathbf{v} \in \mathcal{O}\left(P_{\text {inf }}\right)$. Without loss of generality let $\mathbf{u} \in \mathcal{O}\left(P_{\text {inf }}\right)$. Then note that $g_{r_{\text {inf }}+1}(u)=[1,2 n]$. Clearly $1 \leq \sigma(l(\mathbf{v})) \leq \sigma(r(\mathbf{v})) \leq 2 n$. Therefore $g_{r_{i n f}+1}(u) \cap g_{r_{i n f}+1}(v) \neq \emptyset$.

Subcase 2.2: $\mathbf{u} \notin \mathcal{O}\left(P_{\text {inf }}\right)$ and $\mathbf{v} \notin \mathcal{O}\left(P_{\text {inf }}\right)$. Then either $\sigma(l(\mathbf{v})) \leq \sigma(l(\mathbf{u})) \leq \sigma(r(\mathbf{v}))$ or $\sigma(l(\mathbf{u})) \leq \sigma(l(\mathbf{v})) \leq \sigma(r(\mathbf{u}))$. So $g_{r_{i n f}+1}(u) \cap g_{r_{i n f}+1}(v) \neq \emptyset$.

Therefore $(u, v) \in E\left(I_{r_{\text {inf }}+1}\right)$.

Lemma 4.3. For any $(x, y) \notin E(G), \exists j, 1 \leq j \leq r_{\text {inf }}+1$, such that $(x, y) \notin E\left(I_{j}\right)$.

Proof. Suppose $(x, y) \notin E(G)$.

Case 1: Either $\mathbf{x} \in \mathcal{O}\left(P_{\text {inf }}\right)$ or $\mathbf{y} \in \mathcal{O}\left(P_{\text {inf }}\right)$. Without loss of generality we can assume that $\mathbf{x} \in \mathcal{O}\left(P_{\text {inf }}\right)$. Then $x=w_{i}$, for some $i$ where $1 \leq i \leq r_{\text {inf }}$. In $I_{i}$ as $y \notin N_{G}\left(w_{i}\right)$, we have $g_{i}(x)=g_{i}\left(w_{i}\right)=[0,1]$ and $g_{i}(y)=[2,3]$. Therefore $g_{i}(x) \cap g_{i}(y)=\emptyset$. Hence $(x, y) \notin E\left(I_{i}\right)$.

Case 2: $\mathbf{x} \notin \mathcal{O}\left(P_{\text {inf }}\right)$ and $\mathbf{y} \notin \mathcal{O}\left(P_{\text {inf }}\right)$. As $(x, y) \notin E(G)$ we have either $\sigma(r(\mathbf{x}))<$ $\sigma(l(\mathbf{y}))$ or $\sigma(r(\mathbf{y}))<\sigma(l(\mathbf{x}))$. Therefore $(x, y) \notin E\left(I_{r_{i n f}+1}\right)$.

By combining the above two lemmas we get $E(G)=E\left(I_{1}\right) \cap E\left(I_{2}\right) \cap \cdots \cap E\left(I_{r_{\text {inf }}+1}\right)$. 


\subsection{Tightness result}

Let $G=\overline{\left(\frac{n}{2}\right) K_{2}}$, the complement of the perfect matching on n vertices (We will assume that $\mathrm{n}$ is even). According to the circular arc representation of $\overline{\left(\frac{n}{2}\right) K_{2}}$ described in the introduction, it is easy to see that $\left|\mathcal{O}\left(P_{\text {inf }}\right)\right|=\frac{n}{2}-1$ in $G$. So $b o x(G) \leq \frac{n}{2}$ by Theorem 4.1. But it is known that $\operatorname{box}(G)=\frac{n}{2}$ [7]. So the upper bound for boxicity given in Theorem 4.1 is tight for $\overline{\left(\frac{n}{2}\right) K_{2}}$.

\section{An upper bound based on circular cover number}

Definition 5.1. For a family $F$ of arcs, a circular cover is defined as a subset of arcs of $F$ that can cover the circle. The circular cover number $L(F)$ of the family of arcs $F$ is defined as the cardinality of the minimum circular cover. If there exists no circular cover for $F$, then $L(F)=\infty$.

Definition 5.2. Maximum circular cover number $L_{\max }(G)$ is defined as the maximum value of $L(F)$ obtained over all possible family of arcs $F$ that can represent $G$.

Remark: It may be noted that in many cases getting a circular arc family $F$ that can represent $G$, with higher value of $L(F)$ is preferable. For example see [20], where the upper bound shown for the chromatic number is smaller when $L(F)$ is higher.

Like the parameter $r_{i n f}$, the circular cover number $L(F)$ is also a parameter that appears often in the circular arc graph literature. In this section we will relate $\operatorname{box}(G)$ to the maximum circular cover number $L_{\max }(G)$.

Lemma 5.1. There exists 3 points $P_{0}, P_{1}$ and $P_{2}$ on the circle such that given any vertex $v \in V(G), \boldsymbol{v}$ belongs to at most one of the three sets $\mathcal{O}\left(P_{0}\right), \mathcal{O}\left(P_{1}\right)$ and $\mathcal{O}\left(P_{2}\right)$.

Proof. Let $G$ be a circular arc graph having $L_{\text {max }}(G)>4$. Let $F=\left\{\mathcal{A}_{1}, \mathcal{A}_{2}, \cdots, \mathcal{A}_{L_{\max }(G)}\right\}$ be a minimum circular cover of $G$. Let $\mathcal{A}_{1}, \mathcal{A}_{2}, \cdots, \mathcal{A}_{L_{\max }(G)}$ be the clockwise ordering of the arcs in $F$ (Recall that the clockwise ordering is defined in definition 2.7). Let $l\left(\mathcal{A}_{1}\right)=P_{0}$ and $r\left(\mathcal{A}_{2}\right)=P_{1}$. First we will show that $\mathcal{O}\left(P_{0}\right) \cap \mathcal{O}\left(P_{1}\right)=\emptyset$.

If possible let $\mathcal{A}^{\prime} \in \mathcal{O}\left(P_{0}\right) \cap \mathcal{O}\left(P_{1}\right)$. Then it is easy to see that either we have $\mathcal{A}_{1} \subseteq \mathcal{A}^{\prime}$ and $\mathcal{A}_{2} \subseteq \mathcal{A}^{\prime}$ or $\mathcal{A}^{\prime}$ should contain the portion of the circle that is not covered by $\mathcal{A}_{1} \cup \mathcal{A}_{2}$. In the former case $\left\{\mathcal{A}^{\prime}, \mathcal{A}_{3}, \cdots, \mathcal{A}_{L_{\max }(G)}\right\}$ will be a circular cover of cardinality $L_{\max }(G)-1$, a contradiction. In the latter case $\mathcal{A}_{3}, \cdots, \mathcal{A}_{L_{\max }(G)}$ can be replaced by $\mathcal{A}^{\prime}$ in F. So $\left\{\mathcal{A}_{1}, \mathcal{A}_{2}, \mathcal{A}^{\prime}\right\}$ will be a circular cover of cardinality $3<4<L_{\max }(G)$. Therefore we can infer that $\mathcal{O}\left(P_{0}\right) \cap \mathcal{O}\left(P_{1}\right)=\emptyset$.

Next we will show that any arc passing through $P_{1}$ cannot be clockwise adjacent to any arc passing through $P_{0}$. If possible let some arc $\mathbf{x}$ passing through $P_{1}$ be clockwise adjacent to some arc $\mathbf{y}$ passing through $P_{0}$. Then $\left\{\mathcal{A}_{1}, \mathcal{A}_{2}, \mathbf{x}, \mathbf{y}\right\}$ will be a circular cover of cardinality $4<L_{\max }(G)$, a contradiction.

It follows that there exists some point $P_{2}$ on the circle such that $\mathcal{O}\left(P_{0}\right) \cap \mathcal{O}\left(P_{2}\right)=\emptyset$ and $\mathcal{O}\left(P_{1}\right) \cap \mathcal{O}\left(P_{2}\right)=\emptyset$. Hence the lemma follows.

We shall construct one interval graph corresponding to each of these three points $P_{0}$, $P_{1}$ and $P_{2}$. Let $\Pi_{i}$ be the clockwise ordering of the left and right endpoints of arcs 
starting from $P_{i}$, for $0 \leq i \leq 2$. Note that $\forall \mathbf{u} \notin \mathcal{O}\left(P_{i}\right), \Pi_{i}(l(\mathbf{u}))<\Pi_{i}(r(\mathbf{u}))$ and thus $\left[\Pi_{i}(l(\mathbf{u})), \Pi_{i}(r(\mathbf{u}))\right]$ is a valid interval on the real line. To construct $I_{i}$ we map each vertex $u \in V(G)$ to an interval on the real line by the mapping

$$
\begin{aligned}
g_{i}(u) & =\left[\Pi_{i}(l(\mathbf{u})), \Pi_{i}(r(\mathbf{u}))\right] \quad \text { if } \mathbf{u} \notin \mathcal{O}\left(P_{i}\right) \\
& =[1,2 n] \quad \text { otherwise. }
\end{aligned}
$$

Lemma 5.2. For each interval graph $I_{i}, 0 \leq i \leq 2, E(G) \subseteq E\left(I_{i}\right)$.

Proof. Let $(u, v) \in E(G)$. We have to consider only following two cases

Case 1: When $\mathbf{u} \notin \mathcal{O}\left(P_{i}\right)$ and $\mathbf{v} \notin \mathcal{O}\left(P_{i}\right)$. So either $\Pi_{i}(l(\mathbf{u}))<\Pi_{i}(l(\mathbf{v}))<\Pi_{i}(r(\mathbf{u}))$ or $\Pi_{i}(l(\mathbf{v}))<\Pi_{i}(l(\mathbf{u}))<\Pi_{i}(r(\mathbf{v}))$ which implies that $g_{i}(u) \cap g_{i}(v) \neq \emptyset$.

Case 2: When either $\mathbf{u} \in \mathcal{O}\left(P_{i}\right)$ or $\mathbf{v} \in \mathcal{O}\left(P_{i}\right)$. Without loss of generality let $\mathbf{u} \in \mathcal{O}\left(P_{i}\right)$. Then note that $g_{i}(u)=[1,2 n]$. Clearly $1 \leq \Pi_{i}(l(\mathbf{v})) \leq \Pi_{i}(r(\mathbf{v})) \leq 2 n$. Therefore $g_{i}(u) \cap g_{i}(v) \neq \emptyset$.

Lemma 5.3. For any $(u, v) \notin E(G), \exists i \quad 0 \leq i \leq 2$, such that $(u, v) \notin E\left(I_{i}\right)$.

Proof. In view of Lemma 5.1, at least one of the 3 points $P_{0}, P_{1}, P_{2}$ is such that both the $\operatorname{arcs} \mathbf{u}$ and $\mathbf{v}$ does not belong to the overlap set of this point. Let $P_{i}$ be such that $u, v \notin \mathcal{O}\left(P_{i}\right)$. Without loss of generality let $\Pi_{i}(l(\mathbf{u}))<\Pi_{i}(l(\mathbf{v}))$. Since $(u, v) \notin E(G)$ we can immediately infer that $\Pi_{i}(l(\mathbf{u}))<\Pi_{i}(r(\mathbf{u}))<\Pi_{i}(l(\mathbf{v}))<\Pi_{i}(r(\mathbf{v}))$. Since in $I_{i}$, $g_{i}(u)=\left[\Pi_{i}(l(\mathbf{u})), \Pi_{i}(r(\mathbf{u}))\right]$ and $g_{i}(v)=\left[\Pi_{i}(l(\mathbf{v})), \Pi_{i}(r(\mathbf{v}))\right]$ we have $g_{i}(u) \cap g_{i}(v)=\emptyset$ and therefore $(u, v) \notin E\left(I_{i}\right)$.

Combining Lemma 5.2 and 5.3 we have the following Theorem.

Theorem 5.4. For a circular arc graph $G$, box $(G) \leq 3$ when maximum circular cover number $L_{\max }(G)>4$.

\section{References}

[1] Martin C Golumbic. Algorithmic Graph Theory And Perfect Graphs. Academic Press, New York, 1980.

[2] Naveen Belkale and L. Sunil Chandran. Hadwiger's conjecture for proper circular arc graphs. To appear in European Journal of Combinatorics

[3] Reinhard Deistel. Graph Theory. volume 173, Springer Verlag, New York, 2 edition,2000, 97(5):733-744, September 2007.

[4] Robert B. Feinberg. The circular dimension of a graph. Discrete mathematics, 25(1):27-31, 1979.

[5] E. R. Scheinerman. Intersection classes and multiple intersection parameters. $\mathrm{Ph}$. D thesis, Princeton University, 1984.

[6] Mihalis Yannakakis. The complexity of the partial order dimension problem. SIAM Journal on Algebraic Discrete Methods, 3:351-358, 1982. 
[7] F. S. Roberts. Recent Progresses in Combinatorics, chapter On the boxicity and Cubicity of a graph, pages 301-310. Academic Press, New York, 1969.

[8] J. Kratochvil. A special planar satisfiability problem and a consequence of its NPcompleteness. Discrete Applied Mathematics, 52:233-252, 1994.

[9] M.B.Cozzens and F.S.Roberts. Computing the boxicity of a graph by covering its complement by cointerval graphs. Discrete Applied Mathematics, 6:217-228, 1983.

[10] L.C.Freeman. Spheres, cubes and boxes: graph dimensionality and network structure. Social Networks 5, 139-156, 1983.

[11] R.J. Opsut and F.S. Roberts. On the fleet maintainence, mobile radio frequency, task assignment, and traffic phasing problems in G. The Theory and Applications of Graphs, Wiley New York, 479-492, 1981.

[12] F. S. Roberts. Discrete mathematical models with applications to Social, Biological and Environmental Problems. Prentice-Hall, Englewod Cliffs, New Jersey, 1976.

[13] M.B. Cozzens and F. S. Roberts. Computing the boxicity of a graph by covering its complement by cointerval graphs. Discrete Applied Math. 6 (1983) 217-228.

[14] C. Thomassen. Interval representation of planar graphs. J. Combin. Theory Ser. B 40 (1986) 9-20.

[15] L.S. Chandran and N.Sivdasan. Boxicity and treewidth. J. Combin. Theory Ser. B 97(5) (2007) 733-744.

[16] L.S. Chandran, Anita Das and Chintan D. Shah. Cubicity, boxicity and vertex cover. DOI 10.1016/j.disc.2008.06.003

[17] L.S. Chandran, M.C. Francis and N. Sivdasan. Boxicity and maximum degree. J. Combin. Theory Ser. B 98 (2008) 443-445.

[18] Louis Esperet. Boxicity of graphs with bounded degree. To appear in Europian Journal of Combinatorics.

[19] L.S. Chandran and N. Sivdasan. Geometric representation of graphs in low dimension using axis parallel boxes. Algorithmica DOI 10.1007/s00453-008-9163-5

[20] A. Tucker. Coloring a family of circular arcs. SIAM J. Appl. Math., 29(1975), pp. 493-502. 